\title{
PLAG1: Diagnostic Utility in atypical Pleomorphic Adenoma
}

\author{
Limin Liu*1, Wanlin Xu*2 , Shengwen Liu², Shukun Shen², Francia Fang ${ }^{3}$, Wenjun Yang*2 and Jiang Li*1 \\ ${ }^{1}$ Department of Oral Pathology, Shanghai Ninth People's Hospital, College of Stomatology, Shanghai Jiao Tong University School of \\ Medicine, Shanghai, National Clinical Research Center for Oral Diseases, Shanghai, Shanghai Key Laboratory of Stomatology \& Shanghai \\ Research Institute of Stomatology, Shanghai, China
}

${ }^{2}$ Department of Oral and maxillofacial, head and neck Surgery, Shanghai Ninth People's Hospital, College of Stomatology, Shanghai, Jiao Tong University School of Medicine, Shanghai, National Clinical Research Center for Oral Diseases, Shanghai, Shanghai Key Laboratory of Stomatology \& Shanghai Research Institute of Stomatology, Shanghai, China

${ }^{3}$ Duke University, Durham, NC, USA

${ }^{*}$ Limin Liu and Wanlin Xu contributed equally to this article.

*Corresponding author: Wenjun Yang, Department of Oral and Maxillofacial-Head and Neck Oncology, Shanghai Ninth People's Hospital, Shanghai Jiao Tong University School of Medicine, No. 639 Zhizaoju Road, Shanghai, China

Jiang Li, Department of Oral Pathology, Shanghai Ninth People's Hospital, Shanghai Jiao Tong University School of Medicine, No. 639

Zhizaoju Road, Shanghai, China

\section{ARTICLE INFO}

Received: 慧 March 06, 2019

Published: 絈 March 13, 2019

Citation: Limin Liu, Wanlin Xu, Shengwen Liu, Shukun Shen, Francia Fang, Wenjun Yang, Jiang Li. PLAG1: Diagnostic Utility in atypical Pleomorphic Adenoma. Biomed J Sci \& Tech Res 15(5)2019. BJSTR. MS.ID.002773.

Abbreviations: PA: Pleomorphic Adenoma; PLAG1: Pleomorphic Adenoma Gene 1; LTRs: Long Terminal Repeats; MMTV: Mouse Mammary Tumor Virus;

\begin{abstract}
Pleomorphic adenoma (PA) is the most common type of salivary gland tumor with a high recurrence and malignant transformation. Mounting evidence suggest that pleomorphic adenoma gene 1 (PLAG1) is correlated with the tumorigenesis of pleomorphic adenoma. The PLAG1 transgenic mouse using long terminal repeats (LTRs) of a mouse mammary tumor virus (MMTV) driven transgene method was generated with the intent of exploring distinct mechanisms of PLAG1 gene in pathogenesis of pleomorphic adenomas. Although missing the typical pattern of cartilaginous components, the neoplasm in PLAG1 transgenic mouse model shared the major features when compared to its counterparts of human pleomorphic adenomas. The diagnostic value of PLAG1 was challenged in separating pleomorphic adenoma from other basaloid salivary gland tumors according to previous studies. Here, in order to investigate the detailed patterns of pleomorphic adenoma in PLAG1 transgenic mouse and more importantly, reassure the diagnostic value of PLAG1 in atypical PA, we tested PLAG1 expression and associated markers of the neoplasm in PLAG1 transgenic mouse.
\end{abstract}

Keywords: Pleomorphic Adenoma; PLAG1 Transgenic Mouse Model

\section{Introduction}

\section{Background}

Pleomorphic adenoma (PA), the most common salivary gland tumor, accounts for about $47 \%$ in epithelial tumors of salivary gland tumor and is characterized pathologically by its differentiation in histology and cell phenotypes. Pleomorphic adenoma gene 1, located on 8q12, plays a critical role in tumorigenesis of pleomorphic adenoma, whereas the $t(3 ; 8)(p 21 ; q 12)$ translocation is the most common type $[1,2]$. As a nuclear oncogene, PLAG1 encodes a developmentally regulated transcription factor and constitutes a subfamily of zinc finger proteins [2]. In normal salivary glands, PLAG1 is either not expressed at all or only weakly expressed, in both the acinar and ductal components [3]. The pathological activation of PLAG1 is mainly due to reciprocal chromosome translocation with one of its fusion partners including CTNNB1, CHCHD7, LIFR, TCEA1 [4-7]. The fusion gene leads to up-regulation of PLAG1, which functions as a developmentally regulated transcription factor. The histologic features of pleomorphic adenoma were well 
defined according to the World Health Organization classification of salivary gland tumors.

Typically, a well-circumscribed PA has a broad spectrum of histologic features, which were assessed for myoepithelial cells and luminal epithelial cells, and characteristic. However, PA could be difficult to distinguish from several benign and malignant salivary gland tumors due to its variable morphology. Moreover, the diagnostic utility was challenged when separating PA from other basaloid salivary gland tumors [8]. In human pleomorphic adenomas, the diagnostic value of PLAG1 immunostaining may not be as daunting as we used to believe. However, combining with granular and myoepithelial markers, the immunostaining results could show us a preferential diagnostic landscape of PA. Atypical PA, in PLAG1 transgenic mouse of submandibular perfectly mimic the major pathologic features of human pleomorphic adenoma but cartilaginous components in PLAG1 transgenic mouse. In this study, we explored diagnostic utility of PLAG1 in atypical pleomorphic adenomas in our unique mouse models.

\section{Materials and Methods}

\section{MMTV-PLAG 1 Transgenic Mice}

MMTV-PLAG1 transgenic mice were generated and maintained in C57BL/6 background in Experiment Animal Center, Shanghai Ruijin Hospital affiliated to Shanghai Jiao Tong University, School of Medicine (China). All transgenic mice used in this study were heterozygous for the PLAG1 transgene and were all genotyped via PCR. The entire experimental protocol was approved by the Animal Care and Experiment Committee of Ninth people's Hospital affiliated to Shanghai Jiao Tong University, School of Medicine (Shanghai, China).

\section{Histopathologic Examination and Immunohistochemis- try}

Tissue for histopathologic analysis were rapidly dissected and fixed in 10\% buffered formalin. The tissues were embedded in paraffin and $5 \mu \mathrm{m}$ sections were cut. Sections were stained with hematoxylin and eosin. For immunohistochemistry analysis, sections were deparaffinized through xylene and dehydrated with graded alcohol. Endogenous peroxidase was then blocked with $3 \% \mathrm{H} 2 \mathrm{O} 2$ for $30 \mathrm{~min}$ at room temperature. Antigen retrieval was performed by treating slides with citrate buffer in a microwave for $20 \mathrm{~min}$. The slides were then incubated in moist chamber with first antibody at $4^{\circ} \mathrm{C}$ overnight. After a brief wash in PBS, the slides were treated with a second antibody for $45 \mathrm{~min}$ at $37^{\circ} \mathrm{C}$. The slides were developed in $0.05 \%$ freshly prepared diaminobenze- dine solution (DAB; Sigma, St. Louis, MO) for 5-10 min, then counterstained and mounted. The following antibodies were used: PLAG1 (Novus, Littleton, Colo., USA), cytokeratin 7 (Novus 1:10000,Litttleton,Colo.,USA), cytokeratin 19 (Novus 1:500,Littleton, Colo., USA) calponin (abonova 1:500), SMA (abcam ) and P63 (abcam 1:1200).

\section{Results}

\section{The Pathological Features of Atypical Pleomorphic Adenoma in PLAG1 Transgenic Mouse}

The anatomical site of the neoplasm in PLAG1 transgenic mouse was within the unilateral submandibular gland, completely encapsulated, with multiple-nodules accumulation and clear border with the adjunct normal tissue. Meanwhile, cystic degeneration was scattered in well-demarcated area of PA components. The photomacrograph disclosed the tumor with several tumor bosselations (Figure 1A \& 1B). Some bosselations projected far beyond the range of normal salivary glands, but normally within a complete capsule. Like PA in human salivary glands, PA in PLAG1 transgenic mouse may grow beyond the pseudo-encapsulation, and this feature may not be regarded as invasion (Figure 1C \& 1D). The tumor is pathologically characterized by bland myoepithelial cells in mesenchymal component arranged in short fascicles admixed with tubule-glandular structure (Figure 2A).

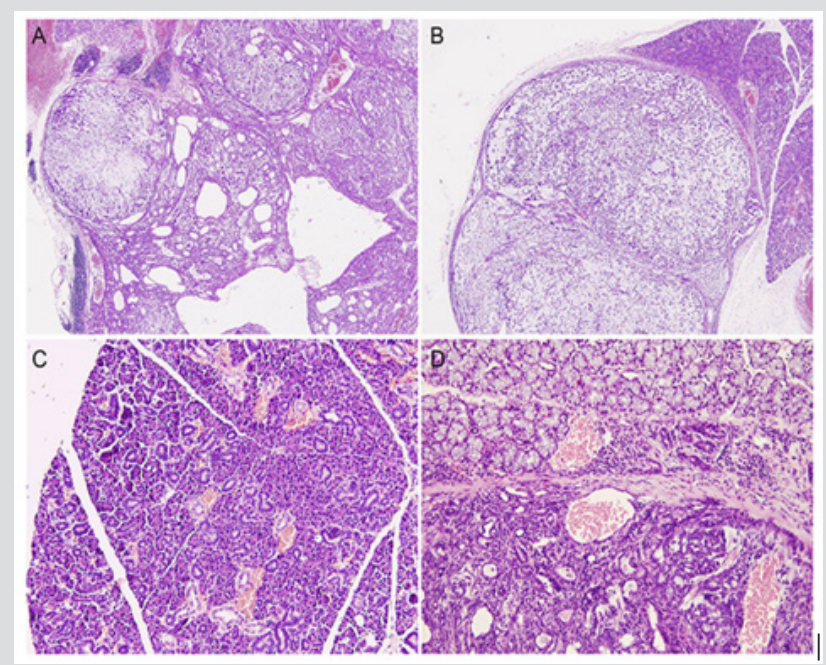

Figure 1: The photomacrograph of the neoplasm in PLAG1 transgenic mouse. (A,B) Neoplasm in PLAG1 transgenic mouse was present with several bosselations. Some tumor bosselations projected far beyond the range of normal salivary glands, but normally within a complete capsule(x200). (C)The normal submandibular gland in PLAG1 transgenic mouse (x400). (D) Neoplasm grew beyond the pseudo-encapsulation $(x 400)$. 
Like human PA, myoepithelial cells were presented in different forms of spindled, plasmacytoid, or polygonal epithelioid myoepithelial cells in PLAG1 transgenic mouse (Figure 2B). Inner abluminal cuboidal cells combined with outer myoepithelial layer exhibited typical luminal-like structure. Hyalinization area with eosinophilic matrix production also displayed the classic pattern of PA (Figure $2 \mathrm{C}$ ). However, chondroid tissue, which is common in human pleomorphic adenoma, was completely absent in this animal model of PLAG1 transgenic mouse. The pathological features of PLAG1 transgenic mouse model was sometimes confused with myoepithelioma. However, the presenting evidence of tubule-glandular structure and myxoid-like tissue can be used to help distinguish PA from its counterparts of myoepithelioma (Figure 2B \& 2D).

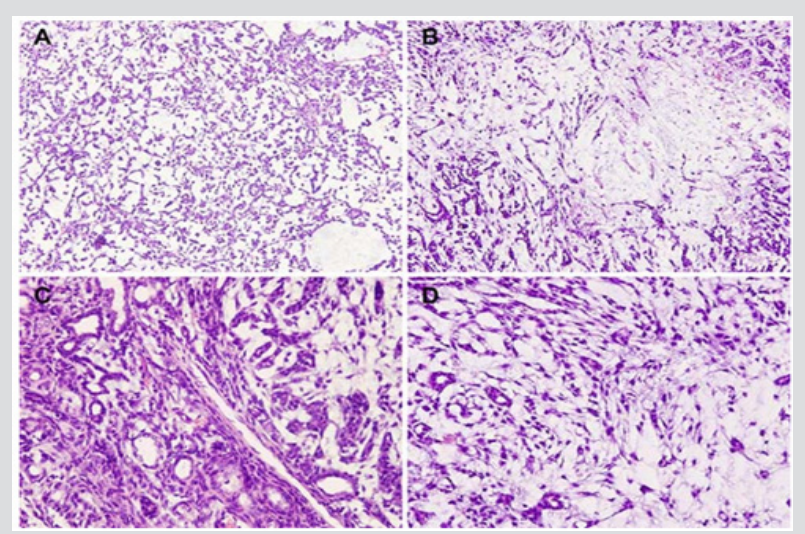

Figure 2: The neoplasm in PLAG1 transgenic mouse exhibited remarkable histopathological similarities to pleomorphic adenoma of human salivary glands. (A) Granular epithelium and myoepithelium (x200). (B) Eosinophilic matrix and Myoepithelial cells of different forms (x200). (C, D) Tubule-glandular structures (x400).

\section{The Diagnostic Utility of PLAG1 in Atypical Pleomorphic Adenoma}

PLAG1, the genetic marker of PA, is positive in lumen-forming cells, tubule-glandular outer layer myoepithelium and stromal spindle cells (Figure 3A). The cytoplasm in most lumen-forming cells showed mild positivity for cytokeratin 7/19 (Figure 3B \& 3C), which verified their nature of granular differentiation. Nucleus of both stromal spindle cells and ductal outer myoepithelium showed strong positivity for p63 (Figure 3D) confirming their squamous or myoepithelial differentiation. Moderate SMA positivity was present mainly in the cytoplasm of ductal outer cells (Figure 3E). Furthermore, there is a strong positivity of S100 and Calponin in the cytoplasm of both stroma spindle cells and ductal outer myoepithelium (Figure 3F \& 3G). The Ki67 proliferation index counts were around 10 to $12 \%$ depending on the field examined (Figure 3H). The morphological patterns together with above immunohistochemical results manifested the diagnostic importance of PLAG1 in atypical pleomorphic adenoma. With a wide morphological spectrum, including cellularity and matrix formation, PA can recapitulate low-grade poly-morphologic tumors of differentiated variants.

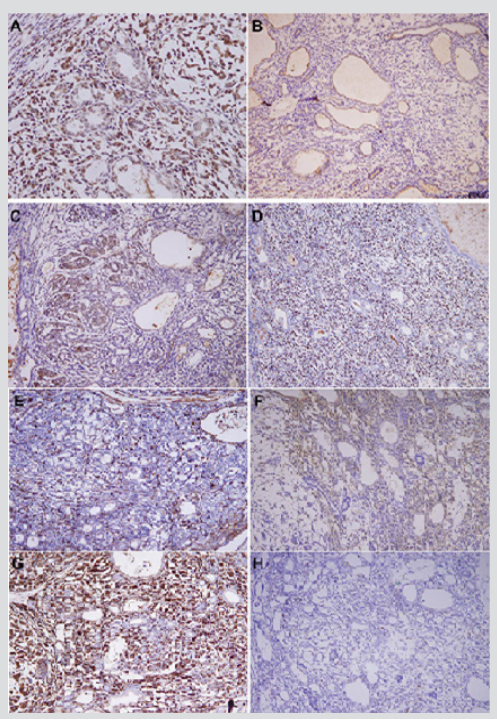

Figure 3: Representative images of the immunohistochemical staining for ranular epithelium and myoepithelial markers. (A) PLAG1 (B) Cytokeratin 7 (C) Cytokeratin 19 (D)p63 (E) SMA (F)S100(G) Calponin (H) Ki67 (x200). 
The diagnostic interpretation of PLAG1 immunostaining is challenging as the expression of PLAG1 in PA is not uniform, while tumor cells with myoepithelial differentiation were immunepositive, those with keratinization were not [8]. In Matsuyama's study, myoepithelial cells showed relatively higher positivity while luminal epithelial cells with keratosic differentiation were less likely to be immunoreactive to PLAG1 [9]. However, our research in this atypical PA shows that both myoepithelial cells and luminal epithelial cells were immunoreactive to PLAG1. In addition, myoepithelial cells show stronger positivity than luminal epithelial cells in this mouse model. On the basis of our knowledge, the genotype is the same in both myoepithelial cells and luminal epithelial cells in pleomorphic adenoma of PLAG1 transgenic mouse. It is possible that there are other mechanisms beyond what we used to think about the regulation of PLAG1. Of interest and in keeping with our prior observation, the cartilaginous component in PA of PLAG1 transgenic mouse was completely absent.

It is likely that the cartilaginous component is derived from an epigenetic mechanism other than PLAG1 overexpression. PLAG1 gene rearrangements are associated with tubule-glandular differentiation and morphologically resembled salivary PA [10]. Concordant with previous studies, our results support the important role PLAG1 plays in the pathogenesis of PA in PLAG1 transgenic mouse. Vaidehi Avadhani et al. found that $72 \%$ cases of PA were immunopositive for PLAG1, and fine-needle aspiration specimens tended to be more immunoactive than surgical excision specimens [8]. In addition, basal cells adenoma fine-needle aspiration specimens and surgical excision specimens of adenoid cystic carcinoma also showed a certain percent of immunoreactive PLAG1 [8]. Thus, immunostaining of granular epithelium and myoepithelial cells were rendered. IHC further aids in establishing both the granular epithelium and myoepithelial nature of the neoplasm in our present study. In PLAG1 transgenic mouse, the neoplasm was strong positive for p63, S100 and calponin but showed mild positivity for cytokeratin 7/19 and SMA.

The neoplasm in PLAG1 transgenic mouse exhibited remarkable histopathological similarities to pleomorphic adenoma of human salivary glands. The unequivocal expression of PLAG1 combined with adjunctive granular epithelium and myoepithelial markers confirmed the final diagnosis and typing. From pathological perspectives, we believe the neoplasm in PLAG1 transgenic mouse perfectly mimics the atypical pleomorphic adenoma of human salivary glands.

\section{References}

1. Kas K, Voz ML, Roijer E, Astrom AK, Meyen E, et al. (1997) Promoter swapping between the genes for a novel zinc finger protein and betacatenin in pleiomorphic adenomas with $\mathrm{t}(3 ; 8)(\mathrm{p} 21 ; \mathrm{q} 12)$ translocations. Nat Genet 15(2): 170-174.

2. Van Dyck F, Delvaux EL, Van de Ven WJ, Chavez MV (2004) Repression of the Transactivating Capacity of the Oncoprotein PLAG1 by SUMOylation. J Biol Chem 279(34): 36121-36131.

3. Rotellini M, Palomba A, Baroni G, Franchi A (2014) Diagnostic utility of PLAG1 immunohistochemical determination in salivary gland tumors. Appl Immunohistochem Mol Morphol 22(5): 390-394.

4. Martins C, Fonseca I, Roque L, Pereira T, Ribeiro C, et al. (2005) PLAG1 gene alterations in salivary gland pleomorphic adenoma and carcinoma ex-pleomorphic adenoma: a combined study using chromosome banding, in situ hybridization and immunocytochemistry. Mod Pathol 18(8): 1048-1055.

5. Asp J, Persson F, Kost Alimova M, Stenman G (2006) CHCHD7-PLAG1 and TCEA1-PLAG1 gene fusions resulting from cryptic, intrachromosomal $8 \mathrm{q}$ rearrangements in pleomorphic salivary gland adenomas. Genes Chromosomes Cancer 45(9): 820-828.

6. Voz ML, Astrom AK, Kas K, Mark J, Stenman G, et al. (1998) The recurrent translocation $\mathrm{t}(5 ; 8)(\mathrm{p} 13 ; \mathrm{q} 12)$ in pleomorphic adenomas results in upregulation of PLAG1 gene expression under control of the LIFR promoter. Oncogene 16: 1409-1416.

7. Astrom AK, Voz ML, Kas K, Roijer E, Wedell B, et al. (1999) Conserved mechanism of PLAG1 activation in salivary gland tumors with and without chromosome 8q12 abnormalities: identification of SII as a new fusion partner gene. Cancer Res 59(4): 918-923.

8. Avadhani V, Cohen C and Siddiqui MT (2016) PLAG1: An Immunohistochemical Marker with Limited Utility in Separating Pleomorphic Adenoma from Other Basaloid Salivary Gland Tumors. Acta Cytol 60(3): 240-245.

9. Matsuyama A, Hisaoka M, Hashimoto H (2011) PLAG1 expression in cutaneous mixed tumors: an immunohistochemical and molecular genetic study. Virchows Arch 459(5): 539-545.

10. Antonescu CR, Zhang L, Shao SY, Mosquera JM, Weinreb I, et al. (2013) Frequent PLAG1 gene rearrangements in skin and soft tissue myoepithelioma with ductal differentiation. Genes Chromosomes Cancer 52(7): 675-682.

\section{ISSN: 2574-1241}

DOI: 10.26717/BJSTR.2019.15.002773

Jiang Li, Wenjun Yang. Biomed J Sci \& Tech Res

This work is licensed under Creative Commons Attribution 4.0 License

Submission Link: https://biomedres.us/submit-manuscript.php

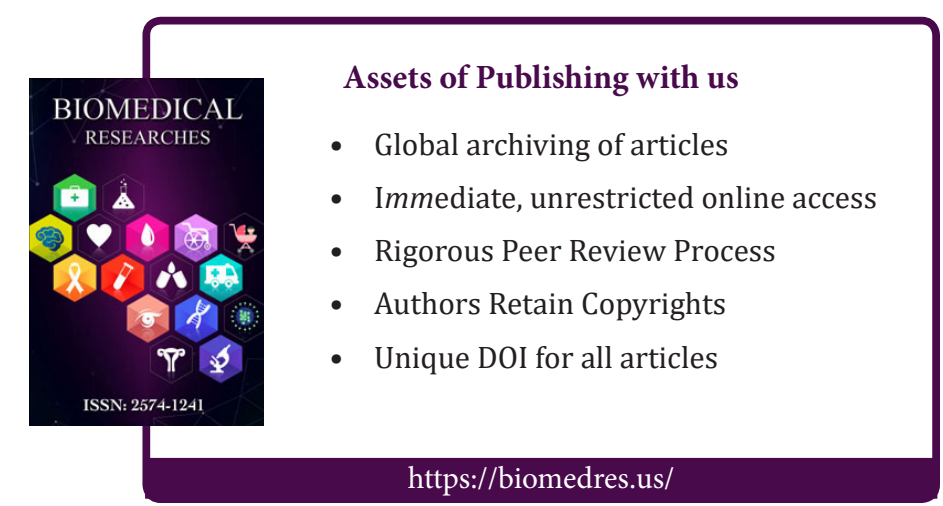

